\title{
A strong clustering of FIR-selected galaxies in the AKARI All-Sky Survey
}

\section{Tomoko Suzuki ${ }^{1,2}$, Tsutomu T. Takeuchi ${ }^{2}$, Agnieszka Pollo ${ }^{3,4}$ and Shinki Oyabu'}

\author{
${ }^{1}$ Department of Astronomical Science, The Graduate University for Advanced Studies, Mitaka, \\ Tokyo 181-8588, Japan \\ email: suzuki.tomoko@nao.ac.jp \\ ${ }^{2}$ Department of Particle and Astrophysical Science, Nagoya University, Furo-cho, Chikusa-ku, \\ Nagoya 464-8602, Japan \\ ${ }^{3}$ National Centre for Nuclear Research, Hoża 69, 00-681 Warsaw, Poland \\ ${ }^{4}$ Astronomical Observatory of the Jagiellonian University, Orla 171, 30-001 Cracow, Poland
}

\begin{abstract}
Various previous galaxy surveys have revealed that different types of galaxies show different spatial distributions. By comparing their clustering strengths, we can investigate the relationship between the environments where galaxies reside and the properties of galaxies. This provides us vital information on when, where, and how galaxies are formed. In this study, we focus on FIR-selected galaxies from AKARI All-Sky Survey, which are considered to be dusty star-forming galaxies. We measure their power spectrum and compare our final result with previous studies using different galaxy samples detected at other wavelengths. It is found that there is the very clear wavelength dependence of the clustering strength.
\end{abstract}

Keywords. galaxies: formation - galaxies: evolution - galaxies: star formation - infrared: galaxies

\section{Results}

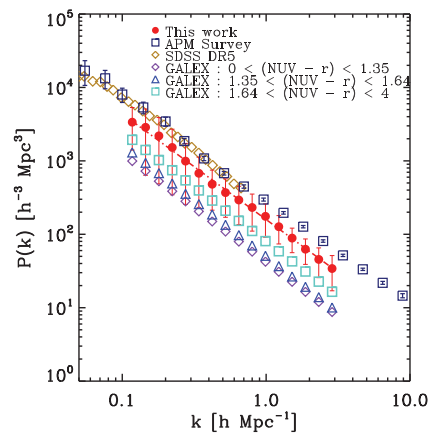

Figure 1. Comparison of our result with power spectra from other galaxy samples.
We obtained 18,077 galaxy candidates from AKARI/ FIS Bright Source Catalog v.1.0 [1]. Our result derived from FIR-selected galaxy sample is shown as red filled circles in Fig.1. It is well approximated by a single power-law and is consistent with the previous study using IRAS PSC $z$ catalog [3]. In Fig.1, we compare our result with three galaxy samples from APM survey [4], SDSS [5], and GALEX [6]. It is found that FIRselected galaxies have lower clustering strength than optical-selected galaxies, but on the other hand, their clustering strength is higher than UV-selected galaxies. FIR-selected galaxies with strong dust emission are expected to be more actively star-forming than UV-selected galaxies on average [7]. Thus, our result suggests that more actively star-forming galaxies distribute in denser regions. This maybe reflecting the spatial propagation of star-forming activities from dense regions to less dense regions, or this maybe due to some external environmental effects inducing dusty star formation in dense environments. As future work, it is necessary to resolve the internal structure of FIRselected galaxies with $\mathrm{AO}$ imaging and/or 3D spectroscopy to investigate the physical processes occurring in each galaxy. 


\section{References}

[1] Yamamura, et al. 2010

[2] Pollo, et al. 2013a, EP\&S, 65, 273

[3] Hamilton, \& Tegmark 2002, MNRAS, 330, 506

[4] Padilla, \& Baugh 2003, MNRAS, 343, 796

[5] Percival, et al. 2007, ApJ, 657, 645

[6] Heinis, et al. 2009, ApJ, 698, 1838

[7] Buat, et al. 2007, ApJS, 173, 404 